\title{
In vitro effect of dexmedetomidine on the respiratory burst of neutrophils
}

\author{
S.L. Chen ${ }^{1}$, W. Zhou ${ }^{2}$, F.Z. Hua', Y. Chen ${ }^{1}$, X.L. Zheng ${ }^{1}$, X.F. Long ${ }^{1}$ and \\ J. Lu ${ }^{1}$ \\ ${ }^{1}$ Department of Anesthesiology, \\ The Second Affiliated Hospital of Nanchang University, \\ Nanchang, Jiangxi Province, China \\ ${ }^{2}$ Department of Vascular Surgery, \\ The Second Affiliated Hospital of Nanchang University, Nanchang, \\ Jiangxi Province, China \\ Correspondence author: J. Lu \\ E-mail: junlu2015@sina.com
}

Genet. Mol. Res. 15 (2): gmr.15028069

Received November 17, 2015

Accepted January 8, 2015

Published June 3, 2016

DOI http://dx.doi.org/10.4238/gmr.15028069

\begin{abstract}
The immunosuppressive effects of dexmedetomidine, a highly selective and widely used $\alpha_{2}$-adrenoceptor agonist for sedation, analgesia, and stress management, are investigated in vitro. In the present study, the respiratory burst of human neutrophils separated from venous blood was evaluated with dexmedetomidine treatment after Escherichia coli stimulation. The effects of five concentrations of dexmedetomidine $(1,5,10,50,100 \mu \mathrm{g} / \mathrm{mL})$ were evaluated by rhodamine in a flow cytometer. The nitric oxide (NO) production and nitric oxide synthase (iNOS) activity were also determined by using commercial kits. The results were compared to the positive control responses (respiratory burst without drug). We found that dexmedetomidine significantly suppressed respiratory burst, NO production, and iNOS activity after stimulation with E. coli, in a dose-dependent manner. The suppressive effects of dexmedetomidine
\end{abstract}


on phagocytic activity of human neutrophils were associated with respiratory burst coupled with $\mathrm{NO}$ production.

Key words: Dexmedetomidine; Respiratory burst of neutrophils; NO production; iNOS activity

\section{INTRODUCTION}

Neutrophils, also known as polymorphonuclear leukocytes (PMN), are the major cell type of the innate immune system. They contain approximately $50-70 \%$ of leukocytes and predominantly eliminate pathogens, inducing acute inflammation (Cowburn et al., 2008; Kantari et al., 2008; Raffaghello et al., 2008). Elimination of pathogens by neutrophils involves a series of physiological processes including chemotaxis, phagocytosis, and microbial killing. The success of pathogen elimination by neutrophils depends on respiratory burst, which is a major process that mediates microbial killing through formation of reactive oxygen species (ROS) and reactive nitrogen species (RNS) (Cowburn et al., 2008). Phagocytosed microbes are internalized into phagosomes, in which ROS are used as microbicides. ROS production is initiated by the NADPH phagosome oxidase (phox) enzyme complex, which produces various types of ROS such as hydrogen peroxide, superoxide anion, oxygen cations, and free radicals (Sheppard et al., 2005). Moreover, RNS results from the catalyst of L-arginine by nitric oxide synthase (NOS) to produce nitric oxide (NO) reactive species. ROS can cause severe bacterial infection when overproduction triggers vascular damage in chronic diseases such as hypertension and arthrosclerosis (Elbim and Lizard, 2009).

Spontaneous activation of neutrophils releases proinflammatory factors and ROS, which exhibits negative effects on vascular tone and their adhesion to the endothelium. Furthermore, a current study has indicated that an excessive load of free radicals secreted by neutrophils at vascular sites aggravated inflammation by limiting the bioavailability of NO secreted by endothelial cells (Chatterjee et al., 2007). Oxidative stress in the neutrophils of hypertensive patients has been shown to associate with increased NADPH oxidase production and lipid peroxidation and decreased cytosolic and mitochondrial superoxide dismutase concentrations (Delles et al., 2008; Hopps et al., 2009; Sedeek et al., 2009). Furthermore, the overexpression of adhesion molecules, such as $\beta_{2}$-integrin, can promote PMN adhesion and leukocyte-endothelium interactions, which may contribute to vascular damage and exacerbate the initiation or complication of arterial hypertension (Tung et al., 2009).

Dexmedetomidine (DEX), a highly selective and potent $\alpha_{2}$-adrenoceptor agonist, is widely used for conscious sedation, analgesia, and stress management in the operating room and the intensive care unit (Chrysostomou and Schmitt, 2008; Zhang et al., 2013). Previous studies have shown that DEX can be administered orally, nasally, transmucosally, or intramuscularly for premedication (Ghali et al., 2011; Özcengiz et al., 2011). However, DEX is not well tolerated hemodynamically (Talke et al., 2003; Farag et al., 2012). High doses of DEX or combinations with other medications often cause bradycardia, hypotension, and serious side effects, which are usually thought to result from the negative feedback modulation of the catecholamine release through the activation of presynaptic $\alpha_{2}$-adrenoceptors expressed throughout the central nervous system (Funai et al., 2014).

Therefore, the main goal of this study was to investigate the respiratory burst activity impairment of DEX in human neutrophils. Respiratory burst was induced from phagocytosis 
of Escherichia coli. An approximate quantity of E. coli was used to produce submaximal stimulation of respiratory burst. Thus, potential suppression or augmentation of the effects of DEX could be verified.

\section{MATERIAL AND METHODS}

After the ethics approval from the Medical Research Ethics Committee of the Second Affiliated Hospital of Nanchang University was received, $5 \mathrm{~mL}$ peripheral blood was drawn from healthy donors $(\mathrm{N}=10)$ and heparinized $(10 \mathrm{U} / \mathrm{mL}$, Liquemin $\mathrm{N}$, Hoffmann-La Roche, Grenzach-Wyhlen, Germany), immediately before the beginning of the experiments.

\section{Tested agents}

DEX was purchased from Waterstone Technology (Carmel, IN, USA). All other chemical reagents used were of analytical grade, and purchased from Sinopharm Chemical Reagent Co., Ltd. (Shanghai, China). Five concentrations $(1,5,10,50$, and $100 \mu \mathrm{g} / \mathrm{mL}$ ) of the tested agents, which were dissolved with phosphate-buffered saline (Dulbecco's without $\mathrm{Ca}^{2+}$ and $\mathrm{MgCl}_{2}$; GIBCO BRL, Eggenstein, Germany), were used for treatment.

\section{Neutrophil isolation}

The following methods were performed to optimize neutrophil isolation. Venous blood $(10 \mathrm{~mL})$ was collected and diluted in 1X Hank's balanced salt solution (HBSS) (Flowlab, Irvine, Scotland) medium at a 1:1 ratio. Diluted blood $(10 \mathrm{~mL})$ was then layered over $5 \mathrm{~mL}$ Lymphoprep (Nycomed, Zürich, Switzerland) and centrifuged at $2000 \mathrm{~g}$ for $40 \mathrm{~min}$ at room temperature. Plasma and the mononuclear cell layer were then discarded. The red cell pellet with PMN and red blood cells (RBC) was suspended in $5 \mathrm{~mL}$ HBSS. The red cell suspension was layered over 3\% Dextran and left at room temperature for 45-60 min to further sediment the RBC. The supernatant from the Dextran sedimentation was collected and the remnant RBC was lysed using a hypotonic lysing procedure to obtain a pure PMN population. Morphological examination and cell count were performed to determine the purity of the PMN.

\section{Sample preparations}

The effects of DEX on respiratory burst were measured by evaluation of the intracellular oxidation of dihydrohodamine 123 (DHR; MoBiTec, Göttingen, Germany) by a flow cytometer. The assay is dependent on the incorporation of DHR into the cell. Subsequently, DEX of different concentrations was added to the samples. After an incubation period of $10 \mathrm{~min}$ at $37^{\circ} \mathrm{C}$, respiratory burst was induced with either $50 \mu \mathrm{L}$ E. coli $\left(1 \times 10^{9} / \mathrm{mL}\right.$, HB 101, Sigma, St. Louis, MO, USA). Stimulation was terminated by transferring the samples onto ice after $20 \mathrm{~min}$. Viability discrimination was performed by adding $10 \mu \mathrm{M}$ propidium iodide (PI; Serva, Heidelberg, Germany) just before measurement. Internal positive controls (submaximal stimulation by E. coli) and negative controls (no stimulation) for each sample were carried out without adding the tested substances. The above-mentioned amounts of $E$. coli that induced submaximal stimulation of neutrophils in the positive control were evaluated in a percentage of inhibition compared to the positive controls in the absence of the tested 
drugs. Pre-stimulation of neutrophils could be excluded by negative controls: respiratory burst without stimuli and agents.

\section{Flow cytometry analyses}

The samples were analyzed using a flow cytometer (FACScan, Becton Dickinson, Heidelberg, Germany). For each sample, 15,000 events were measured. The flow cytometer was equipped with an argon ion laser adjusted to a wavelength of $488 \mathrm{~nm}$. Rhodamine emission was filtered and measured within the spectrum of $515-545 \mathrm{~nm}$ by the corresponding photomultiplier (FL 1). The photomultiplier for FL 3 was used for measuring the PI emission in excess of $650 \mathrm{~nm}$. Forward scatter (FSC), sideward scatter (SSC), and fluorescence signals were adjusted using the negative and positive controls. SSC and the FSC were assessed using the linear mode: FL 1 and FL 3 in logarithmic mode without compensation. Erythrocytes and cell debris were excluded according to a high threshold adjusted in the FSC signals. All of the results were obtained with a constant photomultiplier gain value. Data files were stored in list mode and analyzed in dot plots using the PC-LYSYS software (Becton Dickinson). Neutrophils were included by setting a polygonal gate in FSC versus SSC. These gated cells were transferred to an FL 3/SSC dot plot for exclusion of dead neutrophils due to their high fluorescence in FL 3 as a result of the intracellular PI content. Finally, only vital neutrophils were included in an FL 1/SSC dot plot and the effects of the tested agents on the neutrophil respiratory burst were estimated at approximately 5000 vital neutrophils per sample.

\section{NO production}

The NO production was analyzed by sampling the supernatants after a 12 -h incubation for the presence of nitrite, using the Griess reaction kit (Nanjing Jiancheng, Nanjing, China) according to the manufacturer protocol. Briefly, $100 \mu \mathrm{L}$ supernatant was removed from individual wells and placed on a separate 96-well plate. To each sample, $50 \mu \mathrm{L} 1 \%$ sulphanilamide in $2.5 \%$ phosphoric acid was added, followed by $50 \mu \mathrm{L} 0.1 \% N$-naphthylethylenediamine in $2.5 \%$ phosphoric acid. The optical density of each well was determined at $540 \mathrm{~nm}$. The approximate concentration of nitrite in the samples was determined from a standard curve generated using known concentrations of sodium nitrite.

\section{iNOS activity assay}

Cell lysis buffer ( $1 \mathrm{~mL}$; Beyotime, Suzhou, China) was added to cells cultured in T25 flasks. The supernatants of the cellular lysates were used for iNOS activity assay using an NOS Assay Kit (Nanjing Jiancheng) following the manufacturer protocol.

\section{Statistical analysis}

All statistical analyses were performed with the Statistical Package for the Social Sciences (SPSS) 19.0 software (SPSS Inc., Chicago, IL, USA). The data are reported as means \pm SD from three separate experiments. Statistical significance between two groups (DEX treatment groups versus negative control group) was determined the by paired or unpaired Student $t$-test in cases of standardized expression data. Differences were considered statistically significant at $\mathrm{P}<0.05$. 


\section{RESULTS}

\section{DEX suppressed respiratory burst after stimulation with $E$. coli}

Necrotic neutrophils were excluded due to their high intracellular PI content. The percentage of necrotic neutrophils was under $2 \%$ for all samples. The differences compared to the positive controls without drugs are shown in Figure 1.
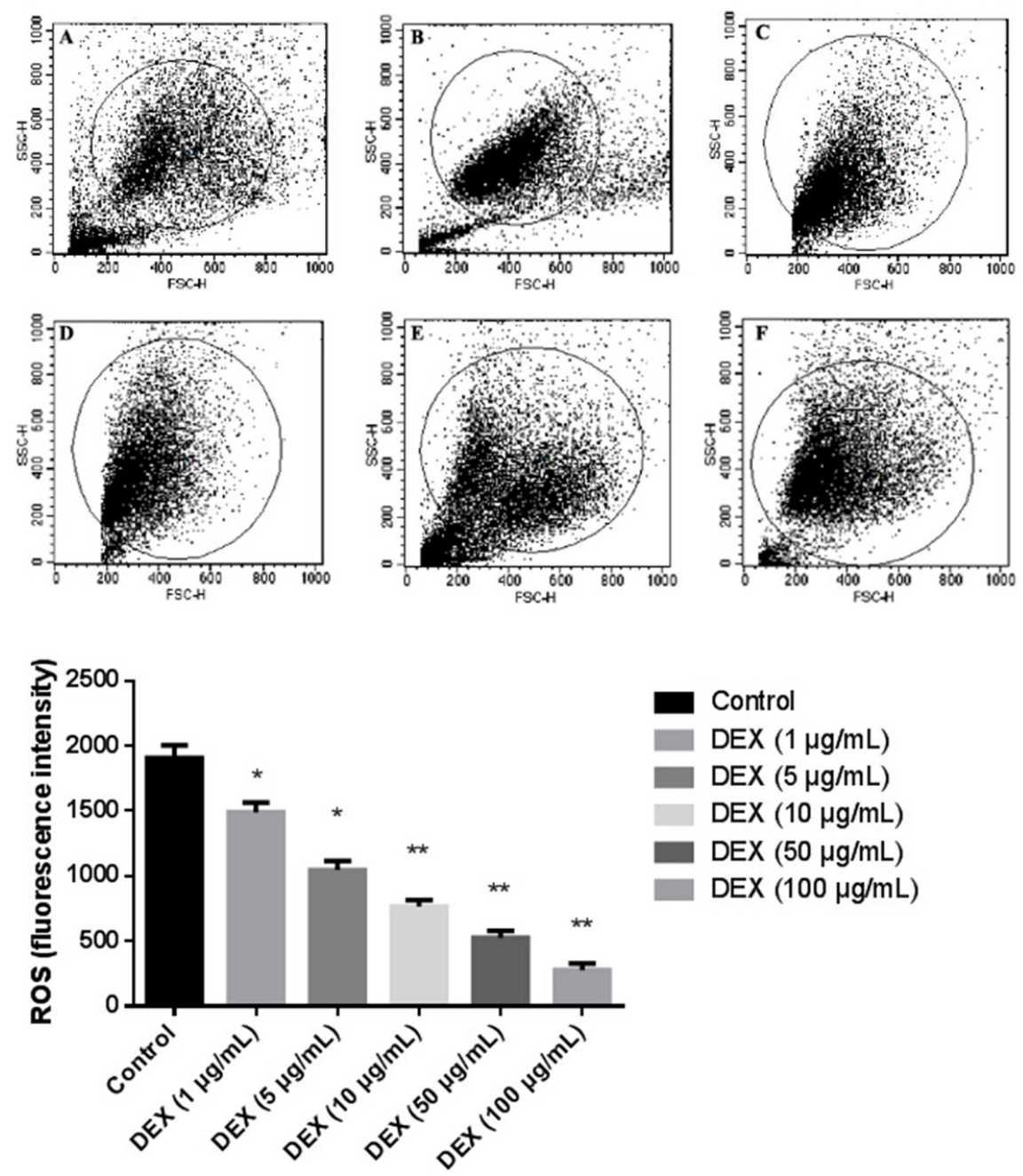

Figure 1. Adjustment of the acquisition dot plots for analysis of the neutrophil respiratory burst due to the green
fluorescence of rhodamine. A. Positive control after Escherichia coli stimulation; B. $1 \mu \mathrm{g} / \mathrm{mL} \mathrm{DEX} \mathrm{after} \mathrm{E.} \mathrm{coli}$ stimulation; C. $5 \mu \mathrm{g} / \mathrm{mL}$ DEX after E. coli stimulation; D. $10 \mu \mathrm{g} / \mathrm{mL}$ DEX after E. coli stimulation; E. $50 \mu \mathrm{g} /$ mL DEX after $E$. coli stimulation; F. $100 \mu \mathrm{g} / \mathrm{mL}$ DEX after $E$. coli stimulation. The bar graphs show, for all experimental conditions, the mean fluorescence intensity, measured by computerized image analysis, reported as means $\pm \mathrm{SD} ; * \mathrm{P}<0.05, * * \mathrm{P}<0.01$ 


\section{Inhibition of NO production}

NO synthesis is catalyzed by iNOS in the presence of superoxide production. Normally, the amount of nitrite is used to represent NO production. Incubation of the neutrophils with DEX has significantly decreased NO production of the cells after stimulation with $E$. coli (Figure 2). Furthermore, the quantity of iNOS at the protein level was determined and the activity of iNOS was measured. The results show that the activity of iNOS was significantly decreased in the DEX-treated neutrophils after stimulation with E. coli (Figure 3).

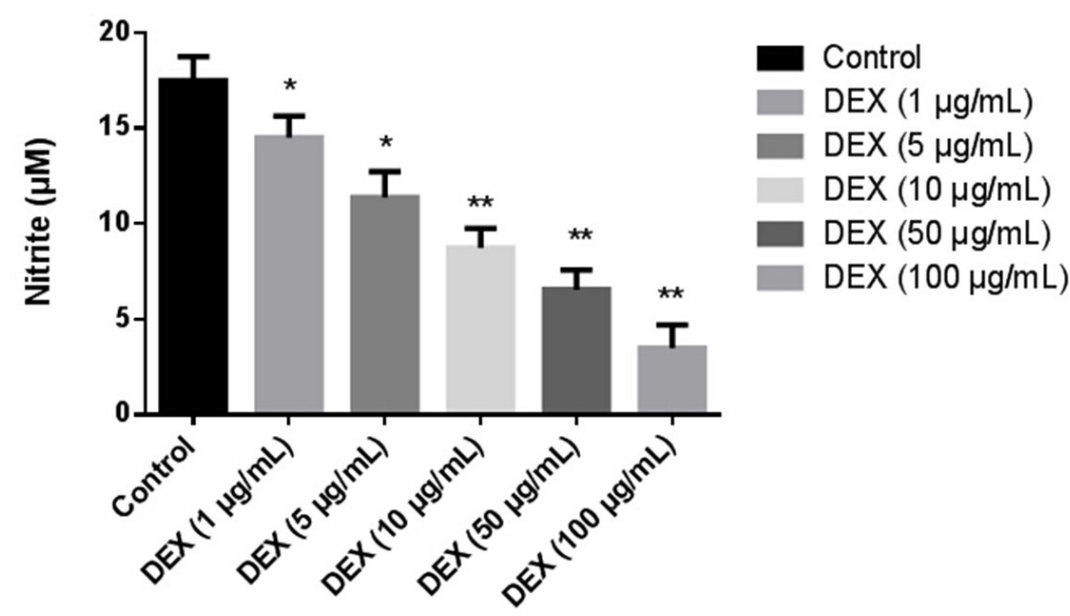

Figure 2. Nitric oxide (NO) production by macrophages incubated with $1,5,10,50,100 \mu \mathrm{g} / \mathrm{mL} \mathrm{DEX}$ after Escherichia coli stimulation, reported as means $\pm \mathrm{SD}, * \mathrm{P}<0.05, * * \mathrm{P}<0.01$.



Figure 3. iNOS activity of macrophages incubated with 1, 5, 10, 50, $100 \mu \mathrm{g} / \mathrm{mL}$ DEX after Escherichia coli stimulation, reported as means $\pm \mathrm{SD}, * \mathrm{P}<0.05, * * \mathrm{P}<0.01$. 


\section{DISCUSSION}

Drugs commonly used in anesthesia for the treatment of sedation, analgesia, and anesthesia are $\alpha_{2}$-agonists (Bergendahl et al., 2004; Constant et al., 2004). Özcengiz et al. (2011) showed that DEX administered orally could prevent post-sevolurane agitation in children. Yuen et al. (2008) reported that $1 \mu \mathrm{g} / \mathrm{kg}$ intranasal DEX produced significantly higher sedation in children aged 2-12 years compared with oral midazolam. The authors emphasized that DEX and midazolam created similar premedication conditions and that both were acceptable (Yuen et al., 2008). In another study, Yuen et al. (2010) found that sedation began an average of 25 min after intranasal administration of DEX and that the mean duration of sedation was $85 \mathrm{~min}$. Sakurai et al. (2010) reported that 3-4 $\mu \mathrm{g} / \mathrm{kg}$ DEX administered to children $1 \mathrm{~h}$ before surgery was reliable and effective.

In a previous study, respiratory burst was stimulated by phagocytosis of $E$. coli or by priming with tumor necrosis factor (TNF)- $\alpha$ followed by receptor activation with the bacterial peptide FMLP. Phagocytosis raised the intracellular $\mathrm{Ca}^{2+}$ content, leading to activation of protein kinase C followed by NADPH oxidase activation (Babior, 1992). Priming with TNF- $\alpha$ was suggested to upregulate neutrophil FMLP receptor expression (Elbim et al., 1993). Priming is defined as the exposure of PMNs to a triggering agent so that there is a markedly increased response to a second stimulus. TNF- $\alpha$ priming is mediated by the $55-\mathrm{kD}$ TNF- $\alpha$ receptor on the surface of neutrophils (Menegazzi et al., 1994). FMLP itself binds to a formyl-peptide receptor, which leads to changes in intracellular calcium concentration followed by oxidase activation (Babior, 1992).

In contrast to the NADPH oxidase, the activity of iNOS is mainly regulated at the transcriptional level (Fang, 2004). Upon the stimulation of microbial pathogens, neutrophils are typically induced to produce ROS immediately. However, RNS production requires de novo protein synthesis (Fang, 2004). ROS and RNS can interact with numerous cellular compounds, including thiols, metals, protein tyrosines, nucleotide bases, and lipids. In the presence of oxygen, $\mathrm{NO} \cdot$ can converted to $\mathrm{NO}_{2} \cdot$ and $\mathrm{ONOO}^{-}$, which can result in oxidative modifications and resemble those that are mediated by ROS alone (Spek et al., 2001). Under certain conditions, the combined antimicrobial activities of ROS and RNS are greater than that of either pathway alone (Pacelli et al., 1995). The synergy of ROS and RNS in the neutrophils stimulated with DEX is not known and needs to be further addressed.

Numerous factors are involved in the regulation of RNS and ROS synthesis, such as proinflammatory cytokines (IFNs, IL-1 $\beta$ and TNF- $\alpha$ ). In our results, DEX was able to reduce the synthesis of ROS and RNS in the neutrophils derived from blood, indicating that the inhibition of ROS and RNS by the neutrophils was associated with lower expression of some proinflammatory cytokines. However, the mechanisms underlying the ROS-RNS inhibition and the lower expression of proinflammatory cytokines by DEX are enigmatic and need to be further characterized.

\section{Conflicts of interest}

The authors declare no conflict of interest.

\section{ACKNOWLEDGMENTS}

Research supported by the Department of Health and Family Planning Committee of Jiangxi Province (Grant \#20123055). 


\section{REFERENCES}

Babior BM (1992). The respiratory burst oxidase. Adv. Enzymol. Relat. Areas Mol. Biol. 65: 49-95.

Bergendahl HT, Lönnqvist PA, Eksborg S, Ruthström E, et al. (2004). Clonidine vs. midazolam as premedication in children undergoing adeno-tonsillectomy: a prospective, randomized, controlled clinical trial. Acta Anaesthesiol. Scand. 48: 1292-1300. http://dx.doi.org/10.1111/j.1399-6576.2004.00525.x

Chatterjee M, Saluja R, Kanneganti S, Chinta S, et al. (2007). Biochemical and molecular evaluation of neutrophil NOS in spontaneously hypertensive rats. Cell. Mol. Biol. (Noisy-le-grand) 53: 84-93.

Chrysostomou C and Schmitt CG (2008). Dexmedetomidine: sedation, analgesia and beyond. Expert Opin. Drug Metab. Toxicol. 4: 619-627. http://dx.doi.org/10.1517/17425255.4.5.619

Constant I, Leport Y, Richard P, Moutard ML, et al. (2004). Agitation and changes of Bispectral Index and electroencephalographic-derived variables during sevoflurane induction in children: clonidine premedication reduces agitation compared with midazolam. Br. J. Anaesth. 92: 504-511. http://dx.doi.org/10.1093/bja/aeh093

Cowburn AS, Condliffe AM, Farahi N, Summers C, et al. (2008). Advances in neutrophil biology: clinical implications. Chest 134: 606-612. http://dx.doi.org/10.1378/chest.08-0422

Delles C, Miller WH and Dominiczak AF (2008). Targeting reactive oxygen species in hypertension. Antioxid. Redox Signal. 10: 1061-1077. http://dx.doi.org/10.1089/ars.2007.2008

Elbim C and Lizard G (2009). Flow cytometric investigation of neutrophil oxidative burst and apoptosis in physiological and pathological situations. Cytometry A 75: 475-481. http://dx.doi.org/10.1002/cyto.a.20726

Elbim C, Chollet-Martin S, Bailly S, Hakim J, et al. (1993). Priming of polymorphonuclear neutrophils by tumor necrosis factor alpha in whole blood: identification of two polymorphonuclear neutrophil subpopulations in response to formyl-peptides. Blood 82: 633-640.

Fang FC (2004). Antimicrobial reactive oxygen and nitrogen species: concepts and controversies. Nat. Rev. Microbiol. 2: 820-832. http://dx.doi.org/10.1038/nrmicro1004

Farag E, Argalious M, Abd-Elsayed A, Ebrahim Z, et al. (2012). The use of dexmedetomidine in anesthesia and intensive care: a review. Curr. Pharm. Des. 18: 6257-6265. http://dx.doi.org/10.2174/138161212803832272

Funai Y, Pickering AE, Uta D, Nishikawa K, et al. (2014). Systemic dexmedetomidine augments inhibitory synaptic transmission in the superficial dorsal horn through activation of descending noradrenergic control: an in vivo patchclamp analysis of analgesic mechanisms. Pain 155: 617-628. http://dx.doi.org/10.1016/j.pain.2013.12.018

Ghali AM, Mahfouz AK and Al-Bahrani M (2011). Preanesthetic medication in children: A comparison of intranasal dexmedetomidine versus oral midazolam. Saudi J. Anaesth. 5: 387-391. http://dx.doi.org/10.4103/1658-354X.87268

Hopps E, Lo Presti R and Caimi G (2009). Pathophysiology of polymorphonuclear leukocyte in arterial hypertension. Clin. Hemorheol. Microcirc. 41: 209-218.

Kantari C, Pederzoli-Ribeil M and Witko-Sarsat V (2008). The role of neutrophils and monocytes in innate immunity. Contrib. Microbiol. 15: 118-146. http://dx.doi.org/10.1159/000136335

Menegazzi R, Cramer R, Patriarca P, Scheurich P, et al. (1994). Evidence that tumor necrosis factor alpha (TNF)-induced activation of neutrophil respiratory burst on biologic surfaces is mediated by the p55 TNF receptor. Blood 84: 287-293.

Özcengiz D, Gunes Y and Ozmete O (2011). Oral melatonin, dexmedetomidine, and midazolam for prevention of postoperative agitation in children. J. Anesth. 25: 184-188. http://dx.doi.org/10.1007/s00540-011-1099-2

Pacelli R, Wink DA, Cook JA, Krishna MC, et al. (1995). Nitric oxide potentiates hydrogen peroxide-induced killing of Escherichia coli. J. Exp. Med. 182: 1469-1479. http://dx.doi.org/10.1084/jem.182.5.1469

Raffaghello L, Bianchi G, Bertolotto M, Montecucco F, et al. (2008). Human mesenchymal stem cells inhibit neutrophil apoptosis: a model for neutrophil preservation in the bone marrow niche. Stem Cells 26: 151-162. http://dx.doi. org/10.1634/stemcells.2007-0416

Sakurai Y, Obata T, Odaka A, Terui K, et al. (2010). Buccal administration of dexmedetomidine as a preanesthetic in children. J. Anesth. 24: 49-53. http://dx.doi.org/10.1007/s00540-009-0863-z

Sedeek M, Hébert RL, Kennedy CR, Burns KD, et al. (2009). Molecular mechanisms of hypertension: role of Nox family NADPH oxidases. Curr. Opin. Nephrol. Hypertens. 18: 122-127. http://dx.doi.org/10.1097/MNH.0b013e32832923c3

Sheppard FR, Kelher MR, Moore EE, McLaughlin NJ, et al. (2005). Structural organization of the neutrophil NADPH oxidase: phosphorylation and translocation during priming and activation. J. Leukoc. Biol. 78: 1025-1042. http:// dx.doi.org/10.1189/jlb.0804442

Spek EJ, Wright TL, Stitt MS, Taghizadeh NR, et al. (2001). Recombinational repair is critical for survival of Escherichia coli exposed to nitric oxide. J. Bacteriol. 183: 131-138. http://dx.doi.org/10.1128/JB.183.1.131-138.2001

Talke P, Lobo E and Brown R (2003). Systemically administered alpha2-agonist-induced peripheral vasoconstriction in humans. Anesthesiology 99: 65-70. http://dx.doi.org/10.1097/00000542-200307000-00014 
Tung JP, Fraser JF, Wood P and Fung YL (2009). Respiratory burst function of ovine neutrophils. BMC Immunol. 10: 25. http://dx.doi.org/10.1186/1471-2172-10-25

Yuen VM, Hui TW, Irwin MG and Yuen MK (2008). A comparison of intranasal dexmedetomidine and oral midazolam for premedication in pediatric anesthesia: a double-blinded randomized controlled trial. Anesth. Analg. 106: 17151721. http://dx.doi.org/10.1213/ane.0b013e31816c8929

Yuen VM, Hui TW, Irwin MG, Yao TJ, et al. (2010). Optimal timing for the administration of intranasal dexmedetomidine for premedication in children. Anaesthesia 65: 922-929. http://dx.doi.org/10.1111/j.1365-2044.2010.06453.x

Zhang H, Zhou F, Li C, Kong M, et al. (2013). Molecular mechanisms underlying the analgesic property of intrathecal dexmedetomidine and its neurotoxicity evaluation: an in vivo and in vitro experimental study. PLoS One 8: e55556. http://dx.doi.org/10.1371/journal.pone.0055556 